\title{
Evolutionary Game Analysis of Fog and Haze Phenomenon
}

\author{
Qingjun $\mathrm{Li}^{1,2, \mathrm{a}^{*}}$ and Xiaoxia Guo ${ }^{3}$
}

\author{
${ }^{1}$ Institute of Science and Technology for Development of Shandong, Jinan, 250014, China; \\ ${ }^{2}$ Soft Science Research Base of Shandong Province and Technology Innovation, Jinan, 250014; \\ ${ }^{3}$ Shandong University of Art \& Design, Jinan, 250014, China; \\ a33104731@qq.com
}

Keywords: Fog and haze phenomenon; Replicator dynamics; Evolutionary game; Strategy

\begin{abstract}
Based on the method of evolutionary game theory, this paper constructs evolutionary game model and analyzes the strategy behavior of bounded rational haze sources. Discussing steady strategy of each haze source when evolutionary game reach stability under different conditions, and proposing some suggestions for governing fog and haze phenomenon.
\end{abstract}

\section{Introduction}

In recent years, fog and haze becomes the main environmental pollution of most cities in our country, it influences more and more cities even some coastal cities, damaging the people's living environment seriously. On the one hand, fog and haze has a negative impact on the environment, and causes great inconvenience to healthy and traveling of people; on the other hand, fog and haze gives rise to great losses for economy of our country, according to statistics, every year economic losses in our country that due to air pollution such as fog and haze is about 1.2\% of GDP [1], it is equivalent to 3.5 times that the expenditure for national medical in China. Therefore, it is time to solve the fog and haze, many scholars made qualitative research on factors [2,3,4], resources, and solving strategies of fog and haze, however, quantitative analysis was lack, and parsing of deeper reasons was not enough. The main problem of hog and haze's emerging is that only considering agent of fog and haze resources' bounded rationality, ignored the solution of fog and haze [5, 6].

For the past few years, evolutionary game is widely applied in various kinds of area [7], this paper is based on the perspective of economics, assuming that the profit-driven side to produce fog and haze is limited rational [8], and possessing the capability to study, imitate, and adjust strategy constantly [9]. Based on the method of evolutionary game [10], this paper provides theoretical references for relative departments of government to make effective solutions about controlling and preventing fog and haze by analyzing the strategy evolutionary process during the parts govern the fog and haze, and revealing the deeper reasons for causing fog and haze.

\section{Evolutionary Game Model of Fog and Haze}

Fog and haze comes from many sources (game party) that are supposed limited rationality in this paper, namely each source can't find the best strategy at the beginning, but can find better strategy through the process of study game; the game would reach equilibrium strategy state when every source gets better strategy, but equilibrium would vary because game party could adjust and improve strategy constantly. In this paper, we assume that existing two sources a and b which are limited rationality in some area, the two sources will make production that can cause fog and haze, sometimes they would govern the fog and haze for production's sustainability.

Therefore, Fig. 1 shows the payoff matrix that describes the strategy environment of sources to participate in game. In Fig. 1, m denotes the expectation payoff that two sources can get when both govern fog and haze; when one source governs fog and haze and the other on not to do, the one to govern gets $\mathrm{n}$ payoff, the other on not to govern gets p payoff; $\mathrm{q}$ denotes the expectation payoff that two sources can get when both not to govern fog and haze. 
Source a

\begin{tabular}{|c|c|c|c|}
\hline & & Govern & don't govern \\
\hline \multirow{2}{*}{ Source b } & Govern & $\mathrm{m}, \mathrm{m}$ & $\mathrm{n}, \mathrm{p}$ \\
\hline & don't govern & $\mathrm{p}, \mathrm{n}$ & $q, q$ \\
\hline
\end{tabular}

Figure 1. Payoff matrix of source

In this paper, all sources in the area are matched randomly in evolutionary game, supposing the rate that sources to govern fog and haze in the area is $x \quad(0 \leq x \leq 1)$, then the rate that sources no to govern is $(1-x)$. Therefore, expectation payoff the two sources can get when taking different strategies and the average payoff of all sources are as follows:

$$
\begin{aligned}
& u_{a}=x \cdot m+(1-x) \cdot n \\
& u_{b}=x \cdot p+(1-x) \cdot q \\
& \bar{u}=x \cdot u_{a}+(1-x) \cdot u_{b}
\end{aligned}
$$

$u_{a}$ denotes the expectation payoff that source a can get when taking strategy to govern fog and haze; $u_{b}$ denotes the expectation payoff that source $\mathrm{b}$ can get when taking strategy not to govern fog and haze; $\bar{u}$ denotes the average payoff that all sources can get in the area. Based on above expectation payoff and related literature, the replicated dynamic equation is as follows:

$$
\begin{aligned}
\frac{d x}{d t}=x\left(u_{a}-\bar{u}\right) & =x\left[u_{a}-x u_{a}-(1-x) \cdot u_{b}\right]=x(1-x)\left(u_{a}-u_{b}\right) \\
= & x(1-x)[x(m-p)+(1-x)(n-q)]
\end{aligned}
$$

when sources play game, one takes strategy to govern whether or not influences the other's expectation payoff necessarily, therefore, the value of $\mathrm{m}, \mathrm{n}, \mathrm{p}, \mathrm{q}$ is usually not equal. For $F(x)=\frac{d x}{d t}=0$ and solving, we can get all stable state of replicated dynamic equation above.

$$
x_{1}^{*}=0, \quad x_{2}^{*}=1, \quad x_{3}^{*}=\frac{q-n}{m-n-p+q}
$$

based on stability theorem of differential equation and characteristic of evolutionary stable strategy, when $F^{\prime}\left(x^{*}\right)<0, x^{*}$ is the evolutionary stable strategy through game, $F^{\prime}(0)=n-q, F^{\prime}(1)=p-m$, $F^{\prime}\left(\frac{q-n}{m-n-p+q}\right)=\frac{(m-p)(q-n)}{(m-p)+(q-n)}$. From the equation above, we find that the relationship of $\mathrm{m}$ and $\mathrm{p}, \mathrm{n}$ and $\mathrm{q}$ both influence expectation payoff of source and the final evolutionary stable strategy. From the above known $0 \leq x \leq 1$, and $u_{a}-u_{b}=x(m-p)+(1-x) \cdot(n-q)$, we discuss the following concentration.

(1) When $m>p, n>q$, under any situation, the payoff source gets if takes governing strategy is always bigger than not to take governing strategy, at this time, the cost source is given a penalty because of not governing is bigger than to pay for governing. At this state, $F^{\prime}(0)>0, F^{\prime}(1)<0$, $x_{3}^{*}>1$ does not meet the stable state conditions, therefore, only $x_{2}^{*}=1$ is evolutionary stable strategy, that is all sources would take governing strategy through playing game repeatedly.

(2) When $m>p, n<q$, under this situation, because speed to study of limited rational sources is slow, under the profit-driven, leading to the later to take strategy will imitate the earlier, at this time, it will emerge two extreme situation, one is that all sources take governing strategy, or all sources not to take governing strategy. At this state, all $x_{1}^{*}, x_{2}^{*}, x_{3}^{*}$ satisfy stable state conditions and $F^{\prime}(0)<0, F^{\prime}(1)<0$, 
$F^{\prime}\left(x_{3}^{*}\right)>0$, therefore, $x_{1}^{*}=0$ and $x_{2}^{*}=1$ are all evolutionary stable strategy. Fig. 2 shows that, when $x \in\left(0, X^{\prime}\right), F^{\prime}(x)<0$, at this time, all limited rational sources will eventually take not governing strategy through playing game repeatedly; when $x \in\left(X^{\prime \prime}, 1\right), F^{\prime}(x)<0$, at this time, all limited rational sources will eventually take governing strategy through playing game repeatedly.

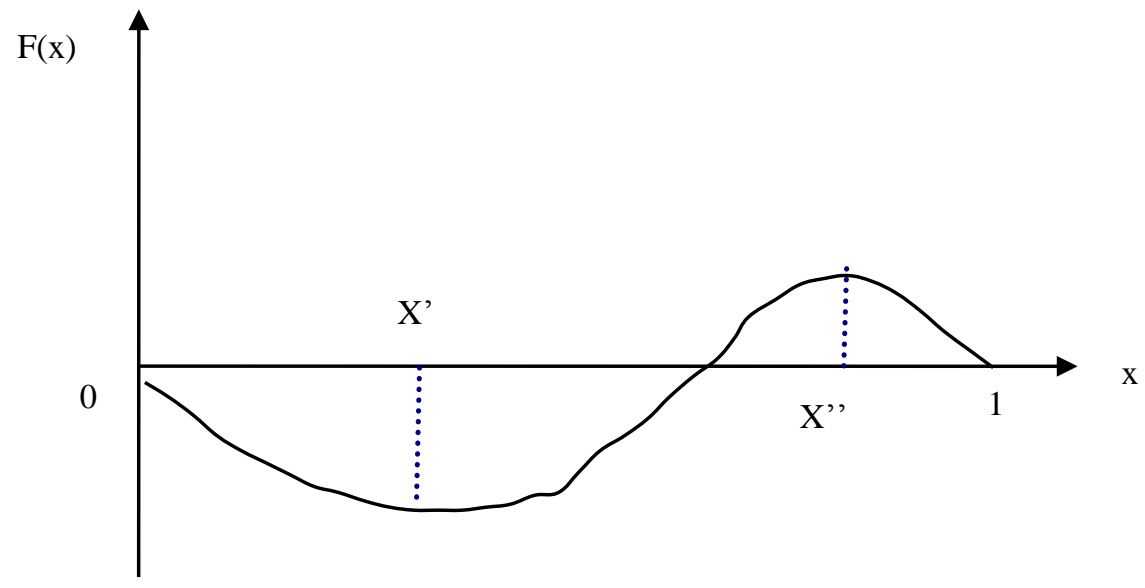

Figure 2. Evolutionary game phase diagram of sources when $m>p, n<q$

(3)When $\mathrm{m}<\mathrm{p}, \mathrm{n}<\mathrm{q}$, under any situation, the payoff source gets if not to take governing strategy is always bigger than taking, at this time, the cost source pays for governing is bigger than given a penalty because of not governing. At this state, $F^{\prime}(0)<0, F^{\prime}(1)>0, x_{3}^{*}>1$ does not meet the stable state conditions, therefore, only $x_{1}^{*}=0$ is evolutionary stable strategy, that is all sources would not take governing strategy through playing game repeatedly.

(4)When $m<p, n>q$, under this situation, if one takes governing strategy, the payoff the other can get when takes governing strategy is smaller than not to take; if one doesn't takes governing strategy, the payoff the other can get when doesn't takes governing strategy is smaller than taking. At this time, for symmetrical playing parties, they will take opposite strategies. At this state, $F^{\prime}(0)>0, F^{\prime}(1)>0$, $F^{\prime}\left(x_{3}^{*}\right)<0$, therefore, only $x_{3}^{*}=\frac{q-n}{m-n-p+q}$ is evolutionary stable strategy. Fig. 3 shows that, when $x \in\left(X^{\prime}, x_{3}^{*}\right), F^{\prime}(x)<0$, at this time, all limited rational sources will eventually take not governing strategy through playing game repeatedly; when $x \in\left(x_{3}^{*}, X^{\prime \prime}\right), F^{\prime}(x)<0$, at this time, all limited rational sources will eventually take governing strategy through playing game repeatedly.

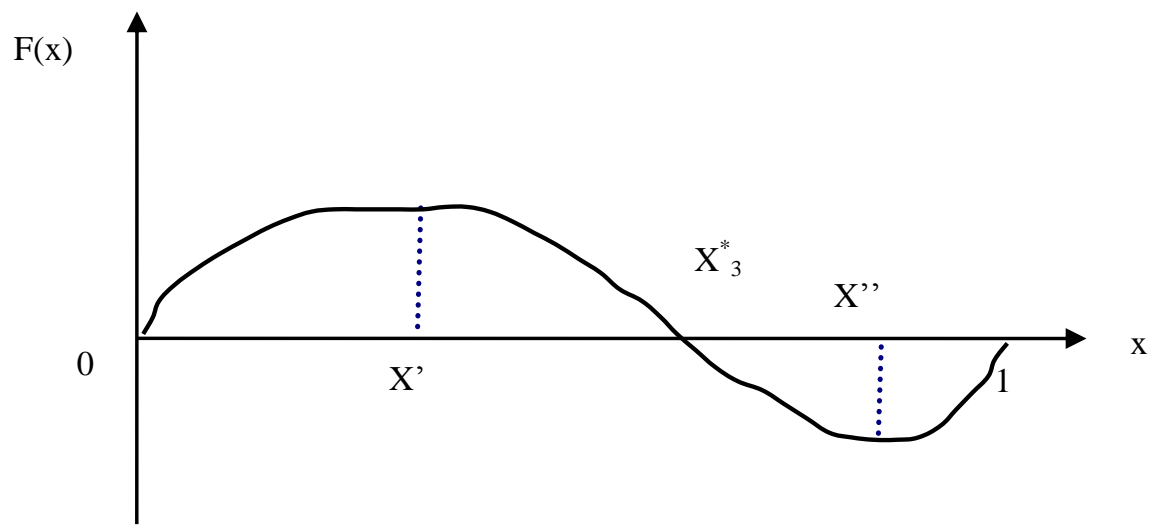

Figure 3. Evolutionary game phase diagram of sources when $\mathrm{m}<\mathrm{p}, \mathrm{n}>\mathrm{q}$ 


\section{Conclusions}

By analyzing the evolutionary of fog and haze above, during playing game repeatedly, sources poss some analytical ability, studying ability and judging ability, one will take strategies that are more profit after observing the others' strategies under the profit-driven; at the same time, environmental protection departments have the responsibility to guide and govern sources, and controlling generation of fog and haze. In conclusion, this paper puts forward the following solutions to govern the fog and haze.

(1) Environmental protection departments must strictly enforce the law, strengthen the level of punishment for sources not to govern. Whether the environmental protection departments perform duty or not will influence the numerical value of $\mathrm{m}, \mathrm{n}, \mathrm{p}, \mathrm{q}$ of this paper, and the behaviors of sources. Let the cost that sources get because of not to govern fog and haze is bigger than to govern, then guiding the source to take governing strategy, and strictly controlling the 'produce' of fog and haze.

(2) Enhancing the levies level of sources. Increasing the cost of sources to produce fog and haze by adding, tax, meanwhile, increasing preferential tax of the green environmental protection unit, strengthening the environmental protection awareness of sources, guiding sources to adjust the form of production, improving industry structure, transforming to green environmental protection unit.

(3) Strengthening the participation and supervision of public. By different Medias to public propaganda let public to know that governing fog and haze is closely linked to our life, and guiding public to actively participate in the governing and controlling of fog and haze; meanwhile, setting report and supervision telephone number that public can use to report and could be rewarded, and then narrowing the space of fog and haze to live. We work together to achieve the state that everybody has responsibility to govern fog and haze, creating a good atmosphere for environmental protection.

(4) Improving the ability of scientific and technological innovation, increasing to explore and use new environmental protection energy. The generation of fog and haze is related to unreasonable energy structure. Our country is rich in new energy such as solar and wind power that we can use to ease our energy crisis, to promote energy structure diversification, decrease the use of high pollution energy, thus reduce fog and haze effectively. For example, the research and use of new energy car can reduce generation of exhaust; wind power generation can reduce the use of high pollution energy such as coal to generate electricity.

\section{Acknowledgements}

This research was financially supported by the Soft Science of Shandong Province for General Projects under grant No. 2014RKC01004 and by Fund for Young Scholars of Shandong Academy of Science under grant No.2014QN041.

\section{References}

[1] Hu Mingwei, The Economic Analysis of the Fog and Haze Phenomenon, J. ECONOMIC RESEARCH GUIDE. 16 (2013) 13-15.

[2] Zhu Chengzhang, Recommended Measures for Controlling Haze Pollution in China, J. SINO-GLOBAL ENERGY. 18 (2013) 1-4.

[3] Lan Xinping, Long Ruyin, A Study on Symbiotic Chain Product Pricing Based on Game Analysis in Coal-Based Cities, J. Resources Science. 9 (2012) 1698-1703.

[4] A. CABRERA, E. F. CABRERA, Knowledge - sharing dilemmas, J. Organization Studies. 5(2012) 687-710.

[5] Lu Fangyuan, Evolutionary Game Analysis on Environmental Pollution Problem, J. System Engineering Theory and Practice. 9 (2014) 148-152.

[6] J. W. WEIBULL, Evolutionary game theory, Cambridge, London, 1995. 
[7] B. Janky, K. Takács, Social Control, Network Structure, and Participation in Collective Action, BME, Budapest, 2005.

[8] McAdam D., Recruitment to High Risk Activism: The Case of Freedom Summer, J. American Journal of Sociology, 1(2013)64-90.

[9] Finkel, S.E, Opp, K.D., Party Identification and Participation in Collective Political Action, J. Journal of Politics, 2(2014)339-371.

[10] Villareal A., Political Competition and Violence in Mexico: Hierarchical Social Control in Local Patrongage Structures, J. American Sociology Review, 4(2011):47-98. 